\title{
Making the List: The Role of Essential Medicines Lists In Reproductive Health
}

After immunization for common childhood illnesses, appropriate use of essential medicines is one of the most cost-effective components of modern health care. ${ }^{1}$ Many interventions for communicable and noncommunicable diseases and conditions that threaten maternal and child health rely on the use of these high-quality medicines. ${ }^{2}$

In 1977, the World Health Organization (WHO) developed its first model list of essential medicines to provide governments with a guide for selecting the drugs and medical devices that best address public health needs. ${ }^{2}$ National essential medicines lists give priority status to the medicines necessary to treat a country's most pressing public health problems. By focusing public sector medicine procurement and treatment on this limited set of essential medicines, resources will be maximized. The lists are, in short, a vital tool for improving and maintaining health. As a result, for more than three decades, WHO has devoted substantial effort to an essential medicines program that seeks to improve access to the most needed drugs and devices.

This comment examines the development of essential medicines lists-both the WHO model list and those compiled by individual countries. We discuss the place devices and medicines for reproductive health occupy on national lists and the importance of their inclusion in reducing the burden of disease attributable to reproductive health problems. We also review the process of establishing essential medicines lists, give examples of their use at the country level, and provide insight into their potential for ensuring access to medicines and devices for reproductive health. Our assessment draws on multiple sources of information, including WHO guidelines on essential medicines, data collected by individual countries, and work completed by WHO, the United Nations Population Fund (UNFPA) and PATH as part of our joint development of a guide to the essential medicines list selection process at the country level.

\section{Determining the WHO Model List}

The idea behind the lists was, and remains, that the use of a limited number of proven, essential medicines leads to better health care, improved supply, lower costs, and broader and more equitable access to products. ${ }^{1}$ Some 30 years later, national essential medicines lists are in place in most WHO member states. ${ }^{2}$ They provide support for national policies regarding the use and availability of medicines and promote primary health care by providing a rationale for the selection and use of medicines and their cost.

Every two years, WHO convenes a committee on the se- lection and use of essential medicines with experts from around the world in such fields as medicine, pharmacology, medicines policy and medicines regulation, as well as other health organizations. The expert committee updates the model list to reflect changes in global health concerns, pharmaceutical developments and patterns of drug resistance. Applications for inclusions, changes or deletions to the model list can be prepared by outside institutions, but must be sponsored or submitted by someone within a WHO program. These applications are intended to be evidence-driven and must explain why a specific drug meets the model list's criteria for acceptance. The criteria take into account disease prevalence, evidence of efficacy and safety, and comparative cost-effectiveness. Applications include the names of the submitting or supporting WHO representative and of organizations consulted in preparing and supporting the application. ${ }^{3}$

The 18th Expert Committee on the Selection and Use of Essential Medicines will be held March 21-25, 2011. The most recent WHO model list (http://www.who.int/ selection_medicines/committees/expert/17/sixteenth_ adult_list_en.pdf) was revised in March 2009 and identifies 340 medicines that address conditions that include malaria, HIV/AIDS, diabetes and cancer. ${ }^{2}$ Organized by category of medication (for example, oxytocics is a category, as is antioxytocics), the model list identifies specific drugs by generic names, such as misoprostol and nifedipine.

Medicines and devices for reproductive health are well represented on the WHO model list. The organization highlighted the importance of reproductive health medicines by issuing The Interagency List of Essential Medicines for Reproductive Health (http://www.who.int/medicines/ publications/essentialmedicines/WHO-PSM-PAR2006\%20I_Rev.pdf) in 2006, the only list devoted to products in a specific field of public health. Much of this comment, however, focuses on the representation of reproductive health medicines on national essential medicines lists.

\section{Compiling a National List}

No public sector or health insurance system can afford to supply or reimburse consumers for the purchase of all medicines that are available on the market. By narrowing the market, essential medicines lists guide countries in the procurement and supply of medicines in the public sector. Essential medicines lists also provide a framework for donors, local pharmaceutical manufacturers and insur-

\section{By Jane Hutchings, Keith Neroutsos and Kathleen Donnelly}

Jane Hutchings is director, Reproductive Health Global Program; Keith Neroutsos is director of procurement, Reproductive Health Global Program; and Kathleen Donnelly is senior publications associate, external relations-all at PATH, Seattle, WA, USA. 
ance systems that reimburse medicine costs. ${ }^{4}$

In addition, national essential medicines lists, together with standard treatment guidelines, serve as the basis for formal education and in-service training for health professionals and for education about medication use for the public. ${ }^{4}$

The WHO model list contains suggestions; each individual country develops its own essential medicines list according to local public health needs. In most countries, the ministry of health appoints a committee to identify medicines for inclusion. ${ }^{4}$ The committee typically includes representation from the ministry, the procurement department, regional and local government health facilities and community health workers.

Manufacturers, nongovernmental organizations, professional associations and advocates, among others, are encouraged to submit evidence-based data on a medicine for consideration. ${ }^{4}$ They may be invited to present their views in an open session, but they do not participate in the decision-making process. The essential medicines list committee also may organize formal and informal consultations with relevant parties, such as representatives of professional organizations, pharmaceutical manufacturers, consumer organizations and the government's budget and finance group.

Essential medicines lists are intended to be aligned with standard treatment guidelines for common health conditions or problems. These guidelines, which are developed in-country and based on global evidence of effectiveness, help standardize treatments throughout a health system and provide reasons and evidence for specific practices. The guidelines include a recommended first-choice treatment, which may be one medicine, several medicines or interventions that do not involve medicines at all. National committees often choose medicines for their lists on the basis of the guidelines' choice of treatment for a specific health condition. ${ }^{4}$ Committees also take into account evidence of the medicine's efficacy and safety in a variety of settings, as well as the relative cost-effectiveness of the medicine, especially when choosing medicines within the same therapeutic category. List committees must also determine that the medicines are available in a form in which adequate quality can be ensured and that the medicines will be stable under anticipated conditions of storage.

A medicine's inclusion on the essential medicines list at the country level does not absolutely ensure that it will be available at all public hospitals and clinics all the time; access to medicines, especially in developing countries, is related to a range of issues. Being on the list, however, does clearly identify the medicine as critical for meeting public health needs and can provide leverage for advocacy if the medicine is not routinely available.

Similarly, a list of essential medicines does not address the questions of who funds the purchase of products or makes the ultimate decision on products for procurement. Essential medicines lists are intended to guide public sector procurement, however, and a medicine's inclusion on the list provides a means of monitoring government spending and a basis for advocating that allocations are made for medicines deemed essential to a country's public health.

\section{How Countries Use Essential Medicines Lists}

As we have noted, adding a medicine or device to an essential medicines list does not guarantee access, nor is it the only action necessary to ensure that these health supplies reach those who need them. The value of essential medicines lists to countries, however, has been demonstrated.

In 2000, for example, the Palestinian Ministry of Health introduced an essential medicines list in an effort to contain costs and improve the use of medicines. ${ }^{5}$ An analysis of prescription patterns between 1997 and 2003 found the essential medicines list effective in improving four prescribing indicators of appropriate use of medicines. The average number of medicines prescribed per clinic visit decreased, as did the percentage of all medicines prescribed that were antibiotics and injections; the percentage of all medicines included in the essential medicines list being prescribed increased by about $5 \%$.

South Africa had no essential medicines policy when the postapartheid government came to power in 1994. At that time, the public sector purchased more than 2,600 pharmaceutical products and there was a strong bias toward tertiary-level medicines. ${ }^{6}$ In 1995, the Minister of Health appointed an essential medicines list committee, and the country's first attempt at such a list was completed and distributed to institutions carrying out public sector procurement the following year. Soon after, an impact study by the South African Drug Action Program assessed the usefulness of the list at primary health care centers. The study selected 30 key medicines to measure the availability of essential drugs, and found that health centers had $85 \%$ of these key drugs available. Of medicines prescribed by health care providers, $70 \%$ were on the essential drugs list, even though there were still medicines not on the essential medicines list in the system. ${ }^{6}$

In India, the national government worked with WHO and the Delhi Society for Promotion of Rational Use of Drugs to launch the Rational Use of Drugs program in 1994. ${ }^{7}$ At the time, the medicine supply in Delhi was erratic. Although the government of Delhi was spending about a third of the health budget on medicines, shortages were chronic. An essential medicines list committee developed a list of 250 essential medicines for hospitals and 100 medicines intended specifically for dispensaries. A common pool for procuring medicines was introduced, and all hospitals managed by the Delhi government began to use the same medicines.

The new measures and bulk buying resulted in a decrease in the procurement prices of essential medicines. ${ }^{7}$ Between 1996 and 2000, the government achieved an estimated savings of $30 \%$ on its annual medicines bill. These savings were used to procure more medicines, which led to an improvement of more than $80 \%$ in the availability of medicines at health facilities. From 1997 to 2002, more than $80 \%$ of prescriptions written by doctors were for 
drugs on the essential medicines list, and patients received $70-95 \%$ of the medicines prescribed to them.

\section{Left Off the List: Reproductive Health}

Too often, national committees compiling essential medicines lists fail to see reproductive health as a national priority, and drugs and devices for reproductive health are left off the list. The reasons behind these omissions are not completely clear, but anecdotal evidence suggests that in some cases, committees focus more on therapeutic medicines and less on preventive medicines, such as contraceptives. As a result, they do not prioritize these supplies for the lists.

In 2003, a WHO report examined 55 national medicine policies and 112 of 192 WHO member countries' national essential medicines lists to determine the degree to which they included reproductive health medicines. ${ }^{8}$ The study compared the medicines found on these essential medicines lists to the WHO 2003 draft Interagency List of Essential Medicines for Reproductive Health. Only two of the national medicine policies surveyed contained any mention of reproductive health.

Furthermore, the study found that across WHO regions, reproductive health medicines and devices were not represented comprehensively on national essential medicines lists, even though solid evidence for their effectiveness existed. ${ }^{8}$ For example, magnesium sulfate, a costeffective medicine used to prevent preeclampsia and treat eclampsia-leading causes of maternal death and illnessappeared on the essential medicines lists of only $40 \%$ of the countries included in the report. On average, only onethird of the contraceptives the report's authors targeted appeared on any one list, and even condoms-essential to preventing pregnancy and the transmission of STIs, including HIV-appeared on only $35 \%$ of the lists examined.

A comparison of the number of medicines included on the model interagency list used in the review and the average number of medicines on national essential medicines lists was similarly lopsided-especially in regard to drugs and devices for HIV/AIDS and family planning. ${ }^{8}$ On average, the national lists included 75 of the 111 products related to safe motherhood and maternal health, 12 of the 22 products for STIs and reproductive tract infections, three of the nine family planning drugs and devices, and five of the 27 drugs for HIV/AIDS on the interagency list.

\section{DISCUSSION}

Reproductive health medicines address a range of health needs, including family planning, prevention of STIs and HIV, and safe pregnancy and delivery. Poor reproductive health accounts for about one-third of the total burden of disease among women of reproductive age and nearly onefifth of the disease burden in the general population. ${ }^{9}$

To alleviate the disease burden within countries, the patterns uncovered by the WHO study must be reversed. Including proven reproductive health medicines and devices on national lists becomes particularly important as fund- ing for global health shifts toward sector-wide approaches. Without strong representation on national essential medicines lists, the visibility and perceived legitimacy of reproductive health medicines and devices will remain low. In turn, the funding needed to purchase and distribute these products will be difficult to attract and maintain, especially as competition for scarce resources increases.

When reproductive health medicines and devices are available, affordable, of good quality and properly used, they can significantly reduce maternal mortality and morbidity and the incidence of STIs. Examples abound:

- Modern contraceptives are highly effective and reduce maternal mortality in three ways. ${ }^{10,11}$ They decrease the total number of pregnancies, each of which puts a woman at risk of death. They prevent unwanted pregnancies that are more likely to end in unsafe abortion. And they reduce the number of births that put women-and newborns-at risk of complications because of the mother's age or the timing between births. It has been estimated that meeting the contraceptive needs of women in developing countries could avert 52 million unintended pregnancies every year, saving the lives of 1.5 million women. ${ }^{10}$

- Postpartum hemorrhage is a major killer of women during pregnancy or childbirth; in Africa and Asia, it is by far the leading cause of maternal mortality. ${ }^{12}$ Oxytocic medicines, including oxytocin, are effective in preventing this serious complication, which affects $1-3 \%$ of all deliveries. ${ }^{13}$

-In women, gonococcal infections can cause long-term complications, such as infertility and chronic pelvic pain, as well as ectopic pregnancy. In nearly all cases, gonococcal infections can be cured with a simple, single-dose regimen of the oral antibiotic cefixime. ${ }^{4}$

Modern contraceptives, oxytocic drugs and cefixime are just three of 16 essential medicines and devices listed in a guidebook developed by PATH, UNFPA and WHO. Essential Medicines for Reproductive Health: Guiding Principles for Their Inclusion on National Medicines Lists (http://www.path.org/publications/details. php?i=1283) is a practical guide to integrating important medicines and devices for reproductive health into national essential medicines lists. The guide was developed to raise awareness among people in the essential medicines community about the importance of reproductive health medicines and to introduce people in the reproductive health community to the potential role of essential medicines programs to help ensure access to reproductive health medicines. Along with the WHO model essential medicines list and The Interagency List of Essential Medicines for Reproductive Health, it provides an overview of the process of and the rationale for including reproductive health medicines on national essential medicines lists.

Not every country needs to include every reproductive drug or device on its national list. But in every country, program managers, policymakers and advocates should understand how medicines are chosen for the list. There are three steps to the process of selection and inclusion in countries with existing lists: 
1. Contact the essential medicines committee and inquire about the revision process.

2. Identify the medicines that should be added to the list, basing choices on the most widespread reproductive health needs.

3. Find reproductive health experts who can provide information to committee members and support adding reproductive drugs and devices to the list.

Evaluations of the guidebook in Malawi and Zambia in 2008 and 2009 indicate that ministry of health staff are aware of the potential that essential medicines lists have to influence and strengthen health care; the evaluations also show that ministry staff are eager to use the lists to improve reproductive health in their countries. In Zambia, reproductive health specialists have compiled an essential medicines list specifically for reproductive health. In Malawi, ministry of health staff are developing the next version of the country's essential health products list, which is slated to include 10 of the 16 reproductive health medicines and devices covered in the guidebook. In both countries, program managers use the list and the guidebook to inform their advocacy work on essential medicines for reproductive health.

Ensuring the inclusion of reproductive medicines on national essential medicines lists is critical, but not the only step in improving access. A recent review of challenges faced by reproductive health programs in six countries suggested several reasons for discrepancies between governmental policies supporting reproductive health and funding allocations for them. ${ }^{14}$ In some cases, the report's authors found, reproductive health-and family planning in particular-had been overtaken by other needs that attracted more external funding. In other cases, governments no longer considered population issues a priority. Finally, the authors noted, some political leaders had become hostile toward certain reproductive health supplies. The designation of a reproductive health medication as essential to the nation's public health, however, provides a platform on which to build advocacy and other strategies to assure access.

\section{CONCLUSION}

The actions in Zambia, Malawi and other countries are encouraging. But they need to be replicated-widely and rapidly-in developing countries across the world.

Political commitment is a first and necessary step toward ensuring the availability of reproductive health products. One way to ensure availability is to mandate that reproductive health medicines are included on essential medicines lists and that the lists are used to guide public expenditures.

Until then, advocating for the inclusion of a comprehensive list of essential reproductive health medicines is important. Improved supply, lower cost and broader access to these medicines holds great promise for improving the health of men and women around the world.

Providing people with information and access to reproductive health medicines that enable them to time and space the births of their children, protect themselves against STIs and make childbirth safer for both mothers and new- borns needs to be a public health priority. The medicines and devices that can help people achieve these goals deserve a place on all nations' essential medicines lists.

\section{REFERENCES}

1. World Health Organization (WHO), The selection of essential medicines, WHO Policy Perspectives on Medicines, Geneva: WHO, 2002, No 4, <http://whqlibdoc.who.int/hq/2002/WHO_EDM_2002.2.pdf> accessed Nov. 18, 2010

2. WHO, Essential Medicines List (EML): 30 years of vital health care, Fact Sheet, Geneva: WHO, 2007, No. 325, <http://www.who.int/ mediacentre/factsheets/fs325/en/>, accessed Apr. 28, 2010.

3. WHO, WHO Medicines Strategy: Revised Procedure for Updating WHO's Model List of Essential Drugs. Report by the Secretariat, Geneva: WHO, 2001, <http://apps.who.int/gb/archive/pdf_files/EB 109/ eeb 1098.pdf>, accessed Nov. 18, 2010

4. Beitz J et al., Essential Medicines for Reproductive Health: Guiding Principles for Their Inclusion on National Medicines Lists, Seattle, WA, USA PATH, 2006, <http://www.path.org/publications/details.php? $\mathrm{i}=1283>$, accessed Nov. 18, 2010

5. Younis MZ et al., Rational use effects of implementing an essential medicines list in West Bank, Palestinian Territories, Expert Reviews, 2009, 9(3):243-250.

6. WHO, South Africa: getting essential medicines to the people, Essential Drugs Monitor, Geneva: WHO, 1999, No. 027, <http://apps. who.int/medicinedocs/en/d/Jhl467e/2.3.html>, accessed Nov. 18, 2010

7. Chaudhury RR et al., Quality medicines for the poor: experience of the Delhi programme on rational use of drugs, Health Policy and Planning, 2005, 20(2):124-136.

8. WHO, Reproductive health medicines in national essential medicines lists: a research report, Background Discussion Paper for the Interagency Consultation on the Selection and Delivery of Essential Medicines and Commodities for Reproductive Health, Geneva: WHO, 2003, No. 3.

9. Singh S et al., Adding It Up: The Benefits of Investing in Sexual and Reproductive Health Care, New York: The Alan Guttmacher Institute 2004, <http://www.guttmacher.org/pubs/archive/addingitup2003. pdf>, accessed Dec. 1, 2010

10. Levine R et al., Contraception, in: Jamison DT et al., eds., Disease Control Priorities in Developing Countries, New York: Oxford University Press, 2006, pp. 1075-1090, <http://www.dcp2.org/pubs/DCP/ 57/FullText>, accessed Nov. 19, 2010.

11. Lule E, Hasan R and Yamashita-Allen K, Global trends in fertility, contraceptive use and unintended pregnancies, in: Lule E, Singh S and Chowdhury SA, eds., Fertility Regulation Behaviors and Their Costs: Contraception and Unintended Pregnancies in Africa and Eastern Europe E Central Asia, Health, Nutrition \& Population Discussion Paper, Washington, DC: World Bank, 2007, pp. 8-39.

12. WHO, Causes of Maternal Death: A Systematic Review, Geneva WHO, 2006, <http://www.who.int/reproductivehealth/topics/ maternal_perinatal/epidemiology/en/index.html>, accessed Nov. 18, 2010 .

13. WHO, Labour, Delivery, and Postpartum, Geneva: WHO, 2008, $<$ http://www.who.int/reproductivehealth/topics/maternal_perinatal/ labour.pdf>, accessed Nov. 18, 2010.

14. Leahy E, Reproductive Health Supplies in Six Countries, Washington, DC: Population Action International, 2009, <http://www. populationaction.org/Publications/Reports/Reproductive_ Health_Supplies_in_Six_Countries/Summary.shtml>, accessed Nov. $18,2010$.

\section{Acknowledgments}

The authors gratefully acknowledge the contribution of Helene Möller, who provided insight into World Health Organization processes and reviewed this comment for technical accuracy.

Author contact: jhutchings@path.org 